\title{
Immunohistochemistry (I.H.C.) and Histopathology of the Fetoplacental Barrier in the Placentas of the Mothers, Recovered from Covid-19 Infection and its Clinical Significance
}

\author{
Satish Patki ${ }^{1 *}$, Rajendra Patil ${ }^{2}$ and Surhud Patki ${ }^{1}$ \\ ${ }^{1}$ Department of Obstetrics and Gynecology, IVF Consultant, Patki Hospital and \\ Research Foundation, Post Graduate Institute of Obstetrics and Gynecolgy, \\ Kolhapur, $M H$, India \\ ${ }^{2}$ Department of Pathology, Consultant Pathologist, Patki Hospital and Research \\ Foundation, Post Graduate Institute of Obstetrics and Gynecolgy, Kolhapur, MH, \\ India \\ *Corresponding Author: Satish Patki, Department of Obstetrics and Gynecology, \\ IVF Consultant, Patki Hospital and Research Foundation, Post Graduate Institute of \\ Obstetrics and Gynecology, Kolhapur, Kolhapur, MH, India.
}

Received: August 26, 2021

Published: September 23, 2021

(C) All rights are reserved by Satish Patki., $\boldsymbol{e t}$

al.

\begin{abstract}
Introduction: Since the outbreak of coronavirus disease 2019 (Covid-19), there has been a continuous research to find out whether the babies born to the affected mothers are infected with Covid-19. Even though, the transmission of this virus has not yet been established by transplacental route, the babies born to covid-positive mothers often show complications like Intra Uterine Growth Restriction (I.U.G.R.) and related problems. Placenta is the most important organ interposed between the mother and fetus and is vital for the fetal health. There are very few studies in the literature about the microscopic functional assessment of the placenta in mothers recovered from Covid-19 infection. Present study is designed to evaluate the biophysical barrier of the syncytium and villous vascularity in the placentas of in such mothers by using Immuno Histo Chemistry (I.H.C.) and histopathology techniques. The microscopic findings are correlated with the perinatal outcome.

Aims and Objective: To study the Immuno Histo Chemistry and histopathology of the syncytium and villous vascularity in the placentas of the mothers, recovered from covid-19.

To study the correlation of these specific findings with the perinatal outcome.

Materials and Methods: Present study was undertaken after getting approval by our Institutional Ethics Committee. The study duration was from Jan 2021 to May 2021. 16 pregnant women who recovered from the proven Covid-19 infection during second trimester by positive RT-PCR test were selected after obtaining informed consent. 15 patients had mild symptoms while one had severe form of Covid-19 infection who required ventilatory support for 5 days. She had intrauterine fetal demise during $30^{\text {th }}$ week of gestation. Age and parity matched 16 patients were selected as controls.

The mothers having hypertension, diabetes and antepartum hemorrhage were excluded. Placentas were collected immediately after removal, washed in tap water and were preserved in $10 \%$ formal saline. Each placenta was cut into three sections, each of 1 $\mathrm{cm}$ thickness, from 3 areas starting form cord attachment to periphery. The tissues were processed and stained by H\&E stain as well as using CD 34 antibody. The slides were examined with light microscope under 10X objective. The number of capillaries per villus was counted in 10 villi in 3 random fields of each slide. Integrity of the syncytium around each villus was also recorded. Perinatal outcome in terms of APGAR score and I.U.G.R. was noted in each case. Data was compared using unpaired t-test with the help of SPSS software version 21 .
\end{abstract}

Citation: Satish Patki., et al. "Immunohistochemistry (I.H.C.) and Histopathology of the Fetoplacental Barrier in the Placentas of the Mothers, Recovered from Covid-19 Infection and its Clinical Significance". Acta Scientific Women's Health 3.10 (2021): 33-37. 
Results: Mean capillary count per villus in the placentas of covid-19 infected patients is found to be significantly low (2.4 +0.5$)$ as compared to that observed in control $(6.4+1)(\mathrm{P}<0.05)$. Majority of the villi in the placenta of the patient who had serious form of covid-19, were completely avascular.

$90 \%$ of the villi of the placentas of control group showed intact layer of syncytium while only $50 \%$ of the villi of the placentas of the patients recovered form covid-19 showed intact syncytium.

APGAR score of the babies of both groups was 8 to 10, however out of 15 babies of the covid-19 recovered mothers, 4 (26.66\%) had I.U.G.R.

Conclusions: Placental syncytial layer constitutes the main interface between fetus and mother. It is exposed to the pathogens, circulating in the maternal blood. Present study has shown that maternal covid-19 infection causes disruption in the integrity of syncytial layer and induces fibrin deposition in the place of syncytiotrophoblast, resulting into reduction in fetal vasculature in placental villi. Even though, vertical transmission of covid-19 infection from mother to fetus is rarely reported in literature, the placental architecture is adversely affected in material covid-19 infection, leading to adverse perinatal outcomes like IUGR, intra uterine fetal death.

As the placentas of women recovered from covid-19 infection show reproducible histopathological abnormalities in villus tree, such women definitely need increased antenatal surveillance. The present study underlines the significance of vaccination against covid 19 infection during antenatal period. We report for the first time such dual study of I.H.C. and histopathology of placentas of mothers recovered from COVID 19 infection in Indian Scenario.

Keywords: Covid-19 Infection; Immunohistochemistry; Histopathology; Placenta

\section{Abbreviations}

P.I.H.: Pregnancy Induced Hypertension; G.D.M: Gestational Diabetes Mellitus; I.H.C.: Immuno Histo Chemistry; RTPCR: Reverse Transcription-Polymerase Chain Reaction.

\section{Introduction}

The placenta is an important interface between the mother and fetus [1], which serves to maintain fetomaternal exchange of blood gases, nutrients and waste [2]. The architecture of the placenta is altered in many non-infective maternal diseases like diabetes [3], hypertension [4] and eclampsia [5].

Since late 2019, Covid-19 has been circulating all over world, infecting more than 70 million people and causing more than 2 million deaths. After the recovery from the first wave of Covid-19 infection, India is facing the second wave. With the observations of increased incidence of I.U.G.R., prematurity and events of intrauterine fetal deaths in the babies born to mothers recovered from Covid 19 infection, we decided to undertake the study of the placentas of such mothers.
The placenta is a temporary endocrine organ which serves two major roles during the course of pregnancy: to nourish and to protect the fetus. The placenta establishes its complex structure throughout the course of gestation. The trophoblastic layer of blastocyst stage embryo differentiates into invasive cytotrophoblasts and multinucleated syncytiotrophoblasts. Invasive cytotrophoblasts penetrate decidua and upper myometrium to establish the firm attachment of the fetus to the mother. Syncytiotrophoblasts form a fused multinuclear surface in the form villi, that is bathed in maternal blood and constitutes the main interface between fetus and mother. Syncytiotrophoblasts are exposed to pathogens, circulating in the maternal blood and appear to have unique resistance mechanism against microbial invasion.

The actual biophysical barrier of the fetus is syncytiotrophoblastic layer on the surface of the placental villi and fetal capillaries inside the villi. The nutrients in the maternal blood are transported through the layer of syncytiotrophoblast to the fetal capillaries which unite finally to form umbilical vein, which takes these nutrients to the fetus. 
In various pathological conditions like pre-eclamsia, the syncytiotrophoblastic layer is affected leading thereby to reduction in the transport mechanism. Due to lack of transport of nutrition and gases, the fetal capillaries undergo degenerative changes and their number is reduced. The reduction in the transport mechanism leads to various fetal disorders like I.U.G.R. or intrauterine death of fetus in extreme cases.

In the present study, we have focused only on this biophysical barrier of syncytiotrophoblastic layer and fetal capillaries by using histopathology and I.H.C. of the placentas of the mothers recovered from Covid-19 infection.

\section{Materials and Methods}

Present study was carried out at Patki Hospital and Research foundation, a Post Graduate Institute of Obstetrics and Gynecology, Kolhapur, MH, India, during the period from January 2021 to May 2021. The study was approved by the Institutional Ethics Committee.

16 pregnant women who recovered from the proven Covid-19 infection during second trimester by positive RT-PCR test were selected after obtaining informed consent. 15 patients had mild symptoms while one had severe form of Covid-19 infection who required ventilatory support for 5 days. She had intrauterine fetal demise during $30^{\text {th }}$ week of gestation. Age and parity matched 16 patients were selected as controls.

The mothers having hypertension, diabetes and antepartum hemorrhage were excluded. Placentas were collected immediately after removal, washed in tap water and were preserved in $10 \%$ formal saline. Each placenta was cut into three sections, each of $1 \mathrm{~cm}$ thickness, from 3 areas starting form cord attachment to periphery. The tissues were processed and stained by H\&E stain as well as using CD 34 antibody. The slides were examined with light microscope under $10 \mathrm{X}$ objective. The number of capillaries per villus was counted in 10 villi in 3 random fields of each slide. Integrity of the syncytium around each villus was also recorded. Perinatal outcome in terms of APGAR score and I.U.G.R. (IntraUterine Growth Restriction) was noted in each case. Data was compared using unpaired t-test with the help of SPSS software version 21.

\section{Statistical analysis}

The data was entered into Microsoft Excel sheet and was analysed using SPSS 21 version software. Continuous data was represented as mean and standard deviation. Independent paired t-test was used as a test of significance to indentify the mean difference between two quantitative variables.

$P$ value of 0.05 was considered as statistically significant after assuming all the rules of statistical tests.

\section{Results}

Mean capillary count per villus in the placentas of covid-19 infected patients is found to be significantly low $(2.4+0.5)$ as compared to that observed in control $(6.4+1)(\mathrm{P}<0.05)$. Majority of the villi in the placenta of the patient who had serious form of covid-19, were completely avascular (Figure 2).

$90 \%$ of the villi of the placentas of control group showed intact layer of syncytium (Figure 1), while only $50 \%$ of the villi of the placentas of the patients recovered form covid-19 showed intact syncytium.

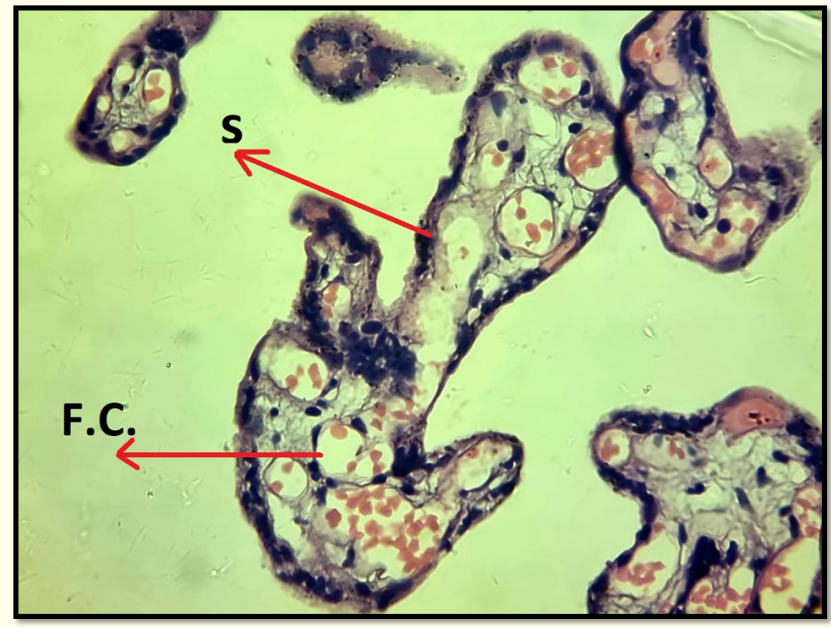

Figure 1: Microphotograph of placental villus showing intact layer of syncitiotrophoblasts (S) and healthy multiple fetal capillaries (F.C) having fetal RBCs in the lumen from the placentas of control group of unaffected patients. 


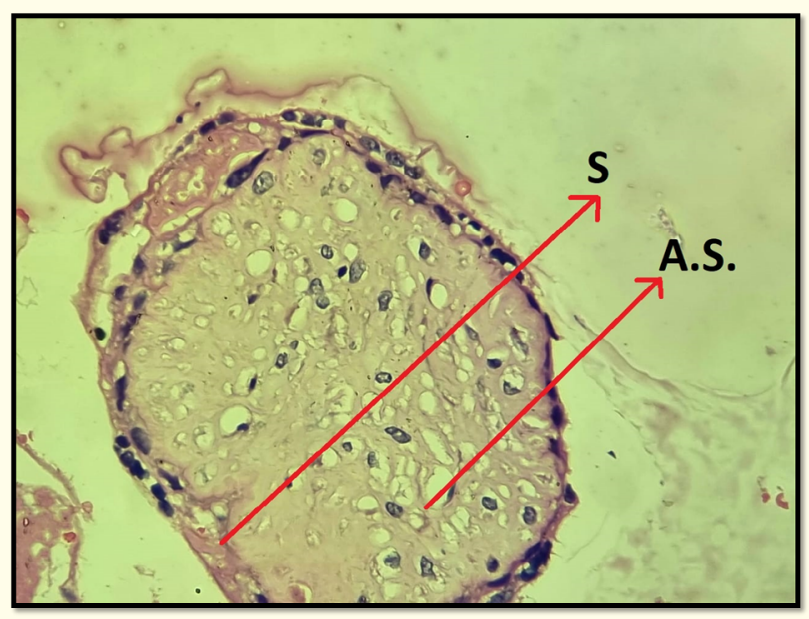

Figure 2: Microphotograph of placental villus showing disrupted layer of syncitiotrophoblasts $(\mathrm{S})$ replaced by fibrin deposition and avascular stroma (A.S.) of the placenta of the mother recovered from Covid-19 infection.

APGAR score of the babies of both groups was 8 to 10 , however out of 15 babies of the covid-19 recovered mothers, 4 (26.66\%) had I.U.G.R. None of the babies born to 16 control group women had I.U.G.R

\section{Discussion}

The placenta is an immunoprivileged organ with attenuated immune response and target of several viral infections [6]. Viruses such cytomegalo virus (CMV), herpes simplex virus (HSV), Rubella Virus, Human Immunodeficiency Virus (HIV) and recently Zika virus have been shown to be able to cross the placental barrier $[7,8]$.

The pandemic of Covid-19 is still in the process of time bound various waves and its pathophysiology is not yet clearly understood till date. None of the studies, have shown the signs of vertical transmission of Covid-19 from mother to fetus [13]. However, Lambelet., et al. [11] have shown the higher rate of miscarriage, intrauterine death and preterm deliveries in mothers recovered after Covid-19 infection. Ng., et al. have documented that, the placental weight was below the 5th percentile in a series of seven such patients [12]. Covid-19 infection is associated with hypercoagulability and changes like thrombi, clots and infarcts in the placental villi along with signs of inflammation are demonstrated in many studies $[6,12]$.
Our study is basically to throw the light on the placental biophysical barrier. $50 \%$ of the villi of the placentas of the patients recovered from covid-19 infection, showed lack of integrity in the syncytium, in the form of fibrin deposition. The mean capillary count per villus in such patients were also significantly low. The lower capillary count is probably the sequeale of the insult of syncytium. Placenta of the patient who had severe form of covid-19 disease, showed absence of capillaries in many terminal villi and hyaline degeneration of the stroma of the villi. Even in the patients who had mild form of disease, the incidence of I.U.G.R. was $26.66 \%$. Placentas of these patients showed signs of fetal malperfusion like avascular villi, karyorrhexis, delayed villous maturation and chorangiosis.

The findings of our study show that there are definite degenerative changes in the placental biophysical barrier of the patients recovered from covid-19 infection. These changes predispose such mothers for the obstetric complication like I.U.G.R. or even intrauterine fetal deaths.

\section{Conclusion}

The result of the present study clearly show that expecting mothers recovered from covid-19 infection should be regarded as high risk pregnant population and should have more surveillance in the form of sonographic monitoring of growth parameters, amniotic fluid volume and Colour Doppler finding of cord flow. The present study also underlines the significance of vaccination against covid-19 as a preventive measure.

\section{Limitations of the Present Study}

Present study is an initial attempt to document the pathology of the placentas of the patients recovered from covid-19 infection. The sample size of the study is limited and has to be widened in future for arriving at more concrete conclusions. Apart from I.H.C. and histopathology modalities, the newer modalities like scanning electron microscopy of the surface of the placental villi will add to our further understanding of the disease at cellular level. Such study is already initiated by our team.

\section{Conflict of Internet}

We declare that there are no conflicts of interest in this research project. There is also no financial support for the project. 


\section{Bibliography}

1. Mardi K and Sharma J. "Histopathological evaluation of placentasin IUGR pregnancies". Indian Journal of Pathology and Microbiology 56 (2003): 551-554.

2. Vogel P. "The current molecular phylogency of Eutherian mammals challenges previous interpretations of placental evoluation". Placenta 26 (2005): 591-5960.

3. Pardo F., et al. "Gestational Diabetes meelitus and the role of adenosine in the human placental endothelium and cetral nervous system". Journal of Diabetes and Metabolism S2 (2012): 010.

4. Barker DJ., et al. "Mechanisms of disease: in utero programming in the pathogenesis of hypertension". Nature Reviews Nephrology 2 (2006): 700-707.

5. Akhlaq M., et al. "Placental morphology in pre-eclampsia and eclampsia and the likely role of NK cells". Indian Journal of Pathology Microbiology 55 (2012): 17-21.

6. MC Smithgall., et al. "Third-Trimester placentas of severe acute respiratory syndrome coronavirus 2 (SARS-CoV-2)-positive women: histomorphology, including viral immunohistochemistry and in-situ hybridization". Histopathology 77 (2020): 994-999.

7. Racicot $\mathrm{K}$ and Mor G. "Risks associated with viral infections during pregnancy". Journal of Clinical Investigation 127.5 (2017): 1591-1599.

8. Lee JK., et al. "Recent Updated on Research Models and Tools to Study Virus-Host Interactions at the Plcenta". Viruses 12.1 (2019): ES.

9. Di Mascio D., et al. "Outcome of coronavirus spectrum infections (SARS, MERS, COVID-19) during pregnancy; a systematic review and meta-analysis". American Journal of Obstetrics and Gynecology MFM 2.2 (2020): 100107.

10. Lambelet V., et al. "SARS-CoV-2 in the context of past coronaviruses epidemics: Consideration for prenatal care". Prenatal Diagnosis (2020): pd.5759.

11. Ng WF., et al. "The placentas of patients with severe acture respiratory syndrome: a pathophysiological evaluation". Pathology 38.3 (2006): 210-218.
12. DA Schwarts. "An analysis of 38 pregnant women with COVID-19, their newborn infants, and maternal-fetal transmission of SARS-CoV-2: maternal coronavirus infections and pregnancy outcomes". Archives of Pathology and Laboratory Medicine (2020).

\section{Volume 3 Issue 10 October 2021}

(c) All rights are reserved by Satish Patki., et al. 